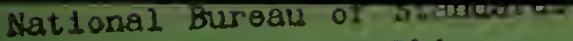

ILibrary, K.W. Bldg

MAR 111965

\section{NBS}

Eechnical Note

\title{
PRACTICAL ASPECTS OF THE USE OF AC-DC TRANSFER INSTRUMENTS
}

E. S. WILLIAMS

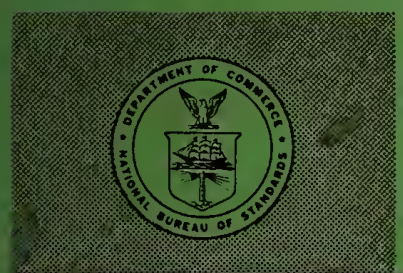

U. S. DEPARTMENT OF COMMERCE NATIONAL BUREAU OF STANDARDS 


\section{THE NATIONAL BUREAU OF STANDARDS}

The National Bureau of Standards is a principal focal point in the Federal Government for assuring maximum application of the physical and engineering sciences to the advancement of technology in industry and commerce. Its responsibilities include development and maintenance of the national standards of measurement, and the provisions of means for making measurements consistent with those standards; determination of physical constants and properties of materials; development of methods for testing materials, mechanisms, and structures, and making such tests as may be necessary, particularly for government agencies; cooperation in the establishment of standard practices for incorporation in codes and specifications; advisory service to government agencies on scientific and technical problems; invention and development of devices to serve special needs of the Government; assistance to industry, business, and consumers in the development and acceptance of commercial standards and simplified trade practice recommendations; administration of programs in cooperation with United States business groups and standards organizations for the development of international standards of practice; and maintenance of a clearinghouse for the collection and dissemination of scientific, technical, and engineering information. The scope of the Bureau's activities is suggested in the following listing of its four Institutes and their organizational units.

Institute for Basic Standards. Electricity. Metrology. Heat. Radiation Physics. Mechanics. Applied Mathematics. Atomic Physics. Physical Chemistry. Laboratory Astrophysics.* Radio Standards Laboratory: Radio Standards Physics; Radio Standards Engineering. ** Office of Standard Reference Data.

Institute for Materials Research. Analytical Chemistry. Polymers. Metallurgy. Inorganic Materials. Reactor Radiations. Cryogenics.** Office of Standard Reference Materials.

Central Radio Propagation Laboratory.** Ionosphere Research and Propagation. Troposphere and Space Telecommunications. Radio Systems. Upper Atmosphere and Space Physics.

Institute for Applied Technology. Textiles and Apparel Technology Center. Building Research. Industrial Equipment. Information Technology. Performance Test Development. Instrumentation. Transport Systems. Office of Technical Services. Office of Weights and Measures. Office of Engineering Standards. Office of Industrial Services.

* NBS Group, Joint Institute for Laboratory Astrophysics at the University of Colorado.

** Located at Boulder, Colorado. 


\title{
NATIONAL BUREAU OF STANDARDS Technical Note 257
}

ISSUED MARCH 9, 1965

\section{PRACTICAL ASPECTS OF THE USE OF AC-DC TRANSFER INSTRUMENTS}

\author{
E. S. Williams
}

NBS Technical Notes are designed to supplement the Bureau's regular publications program. They provide a means for making available scientific data that are of transient or limited interest. Technical Notes may be listed or referred to in the open literature. 
CONTENTS

PAGE

1. GENERAL-

2. AC-DC DIFFERENCE-

3. GALVANOMETER CALIBRATION-

4. A-C MEASUREMENTS- -5

5. PRECAUTIONS-

6. SAMPLE CALCULATIONS---

\section{LIST OF FIGURES}

1. Circuits for AC-DC Difference Measurements

(a) for voltage, (b) for current

2. Galvanometer Scale Calibration

3. Circuits for AC Voltage Measurements

4. Circuits for AC Current Measurements 


\author{
PRACTICAL ASPECTS OF \\ THE USE OF AC-DC TRANSFER INSTRUMENTS \\ E.S。W111iams
}

E1ectrothermic transfer instruments may be used to make direct measurements of ac-dc difference or frequency influence. With a somewhat similar procedure an "unknown" a-c voltage or current may be accurately measured by determining the difference between it and a preset and accurately measured d-c equivalent. Test circuits and procedures are described and data taking and calculations are illustrated.

\title{
1. GENERAL
}

The term "transfer instrument" is the name used here to designate an instrument used to transfer direct current standards to alternating quantity measurements. It is analogous in a way to the chemist's balance. It compares a direct current or voltage established directly in terms of a basic standard with the similar a-c quantity to be measured. In use it is ordinarily transferred---or switched---between $a-c$ and $d-c$ circuits. The primary considerations in its design, therefore, are not convenience nor direct readability but high sensitivity of readout or indication and small ac-dc differences.

The thermoelement is the current sensing element in the transfer instruments to be considered here. The short straight heater gives it minimum reactance and makes it an excellent component in a circuit where a very small frequency influence is important. The thermocouple is attached to the mid-point of the heater and is insulated from the a-c circuits by a small ceramic bead. For currents up to one ampere the assembly is mounted in an evacuated glass bulb. Thermoelements for currents higher than one ampere usually do not have insulating beads or vacuum enclosures.

For current measurements, the transfer instrument may cons is $t$ simply of a single thermoelement whose heater carries all the current 
to be measured, or it may consist of a thermoelement in parallel with a-c shunts。 For voltage measurements, the thermoelement usually carries only a few milliamperes. It is connected in series with a multiplier resistor which is very often tapped to give several voltage ranges, although single-range transfer voltmeters are widely used at higher frequencies.

AC-DC difference tests are often used to advantage. Electrodynamic and electrothermic instruments respond equally to $d-c$ and 1 ow-frequency a-c currents. They are more readily calibrated with d-c voltage or current using the conventional $\mathrm{d}-c$ techniques---the potentiometer, volt box, and shunt. Since the scale accuracy depends on the accuracy of series multiplier resistors or shunts, it is subject to changes or longtime drifts, and frequent recalibration with direct current is advisable.

On the other hand the ac-dc difference or frequency influence is much more constant. It is the result of residual reactance in the a-c circuit. If the instrument is well designed, and constructed, so that multiplier, shunt and coil components are rigidly mounted, there should be no appreciable change in the. frequency characteristics unless these parts are repaired or altered. Ordinarily ac-dc difference corrections are redetermined only once in several years and, if significant, are applied in addition to scale calibration corrections determined in $\mathrm{d}-\mathrm{c}$ tests.

This calibration scheme has the advantage of separating the types of errors. It simplifies the maintenance of the calibration since the more difficult a-c techniques are required infrequently.

Many important instruments, of course, cannot be standardized in this way. Among these are instruments with built-in transformers and many types of moving-iron instruments, vacuum tube voltmeters, rectifier instruments, and some multirange electrostatic voltmeters. These usually require direct measurements of a-c quantities, but a digital instrument or a volt-ampere converter having an accuracy of 0.03 to 0.05 percent is usually suitable for the calibration.

There is, however, increasing reliance on a-c digital voltmeters and $a-c$ voltage standards of rather high accuracy and reliability. If these are to be tested with appropriate accuracy, they must be compared as directly as possible with the best $d-c$ standards. The ac-dc transfer instrument is the comparator. But care must be taken to avold inaccuracies that can arise from drift, $d-c$ reversal difference, and other causes if we are to realize its full potential accuracy.

\section{AC-DC DIFFERENCE}

Figure la shows a multirange transfer voltmeter and the circuits for an ac-dc difference test. Instead of the usual millivoltmeter 
connected to the thermocouple output, as in electrothermic indicating instruments, the transfer instrument has a Iindeck potentiometer or other balancing circuit. This 18 adjusted to equal or balance the thermocouple output. A galvanometer null indleates the balance.

To make an ac-dc difference or frequency influence test on the "TEST Vm" (it may be an indicating instrument or another transfer instrument with its Lindeck potentiometer and galvanometer) it is connected in parallel with the transfer instrument as shown so both instruments can be switched to $a-c$ or $d-c$ voltage (AC-V or DC-V, Fig. 1a). The test voltmeter is set to a certain test point on d-c voltage and the Inindeck potentiometer is adjusted so that the galvanometer is balanced near midscale. This need not be a null balance. Then, without further Lindeck adjustment, the test and transfer voltmeters are switched to a-c and then to $\mathrm{d}-\mathrm{c}$ voltage. The test instrument is set to the same test point in each case, and the galvanometer indications corresponding to the two voltages are read. The difference in the indications is a measure of the difference between the $a-c$ and $d-c$ voltages required to give equal deflection or response to the instrument under test.

The transfer instrument usually has two shortcomings which must be taken into account for highest accuracy. The thermoelement has a small reversed $d-c$ difference (that is, the emf output is not quite the same for equal heater current in opposite directions) and it frequently has a drift in output emf even after several minutes of warm-up. The drift is particularly noticeable on higher voltage ranges where there is considerable heating in the multiplier resistors.

To overcome these two difficulties, the following procedure is recommended: after an initial galvanometer reading on a-c voltage (AC-V) we take two on DC-V---one for each direction of current---and then, In case there is a drift, a fourth reading on $\mathrm{AC}-\mathrm{V}$. If the drift rate is reasonably uniform, as it usually is after a short warm-up, and if the readings are taken at rather regular time intervals, the effect of drift can be practically eliminated. Even with a large drift the determinations can be repeated surprisingly well with this procedure.

An alternative procedure is to take readings on $\mathrm{DC}-\mathrm{V}, \mathrm{AC}-\mathrm{V}$ and reversed $D C-V$. This w11I also compensate for drift, but a second $A C-V$ reading is preferred as a check on the first one---as a precaution.

For current measurements (Fig. 1b) the transfer instrument--in this case a single thermoelement--has its output connected to the same Lindeck potentiometer and galvanometer as that shown in Fig. 1a. Again the "TEST $\mathrm{Am}^{\prime \prime}$ may be an indicating instrument or a transfer instrument. The procedure described above for voltmeters is also used here.

The ac-dc difference is indicated in terms of galvanometer scale divisions ( $\mathrm{cm}$ or $\mathrm{mm}$ ). We therefore need a calibration of the galvanometer 
scale for each thermoelement to be used. If the calibration is made and a curve drawn as described below, then a dividing factor may be found to conveniently convert scale divisions to percent difference.

\section{GALVANOMETER CALIBRATION}

Before making the galvanometer scale calibration (or using it in an instrument test) the galvanometer must be connected so an increase in heater current gives an upscale deflection or a deflection to the right on the galvanometer scale. This is important to assure a proper sign with the data in Instrument calibration.

Figure 2 shows, on the left, a simple circuit by which we can pass a direct current through the heater of the thermoelement, and a shunt and a potentiometer to measure the current. Again the output emf of the thermocouple is balanced against the Lindeck potentiometer as indicated by the galvanometer. Actually this balance should be such that the galvanometer deflects a little to the left of mid-scale. Having measured the current accurately, we then accurately measure an increased current $\Delta I$ of perhaps 1 percent. This increase in current will deflect the galvanometer upscale (beyond mid-scale) and this change in deflection we designate $\Delta D$ 。

From the denominator terms of the equation (Fig. 2), we see that this $\% \Delta I$ gives an increased deflection $\Delta \mathrm{D}$. Now another change in current which we designate in the numerator as \%DIFF (or more exactly, a small difference between $a-c$ and $d-c$ currents) will then give another change in deflection which we designate $\mathrm{D}_{\mathrm{ac}}-\mathrm{D}_{\mathrm{dc}}$. This gives us a simple ratio

$$
\frac{\% D I P F}{\% \Delta I}=\frac{D_{a c}-D_{d c}}{\Delta D}
$$

If $\Delta I$ was one percent, the denominator term in the first expression $(\% \Delta I)$ is unity, and the percent difference between the $a-c$ and $d-c$ current (or voltage) is given by the equation $\% \mathrm{DIFF}=\left(\mathrm{D}_{\mathrm{ac}}-\mathrm{D}_{\mathrm{dc}}\right) / \Delta \mathrm{D}$ and $\Delta D$ is a sensitivity factor which we may call $\mathrm{S}$. The complete formula including the percentage ac-dc difference correction $\left(C_{g}\right)$ for the transfer instrument is

$$
\% D I F F=\frac{D_{a c}-D_{d c}}{S}+C_{s} \text {. }
$$

The calibration may be made with larger or smaller $\Delta I$, but $S$ should be equal to the $\Delta D$ obtained with one percent $\Delta I_{\text {o }}$ That is, if $\Delta I$ is $2 \%$ then $S=\Delta D / 2$ (see sample calculation). A one-point calibration is, of course, good only at that particular current level. We have found it convenient to make four or more determinations and to draw a curve connecting each point as shown in Fig. 2. (Values of "TC mV" and "S" 
on the figure are used in the sample calculations, page 9.) We then find a value of $S$ corresponding to any current level or thermocouple output emf. If the thermocouple output is measured with the Lindeck potentiometer, it is convenient to use it as the abscissa in drawing and using the curve. Otherwise, one may use heater current or percentof-rated-voltage as abscissa.

The curve is not quite straight so that one numerical factor cannot be used to accurately determine S from TC mv directly. The error would sometime be 20 percent or more at the upper end of the curve.

The curve is easily determined and drawn and is reliable until the thermoelement is replaced if the circuit components and galvanometer sensitivity are unchanged. The galvanometer should be checked now and then to see that its sensitivity is unchanged. The scale should also be reasomably linear over the portion being used. We have found that most galvanometers are satisfactory in this respect and that sensitivity rarely changes.

We should also note that where the differences being measured are sma11, we can tolerate surprisingly large inaccuracy in the galvanometer and its calibration. For instance, if we have a 10 percent error in a measurement of a 0.1 percent voltage difference, we are wrong by only 0.01 percent of the total voltage.

This calibration curve may be made for a transfer voltmeter on any range and the voltage and voltage change $(\Delta V)$ can be measured with a volt box and potentiometer (see sample calculation). Also if a calibrated d-c supply is available with which small incremental changes can be set by decade switching, it can be used to make the galvanometer scale calibration.

Incidentally, some commercially available transfer voltmeters now have built-in arrangements for determining galvanometer sensitivity。

\section{A-C MEASUREMENTS}

With the galvanometer scale calibrated, we are also prepared to make accurate $a-c$ voltage and current measurements. Figure 3 shows the circuit for voltage. The a-c source shown on the left may be the voltage applied to a deflecting or digital voltmeter or it may be the output of a calibrated a-c voltage standard. The transfer voltmeter can be switched back and forth between the $a-c$ and $d-c$ voltages with its output connected to the Lindeck potentiometer and galvanometer. The d-c voltage supply is shown on the right with the reversing switch and the volt box and potentiometer.

A measured d-c voltage could be supplied, of course, by a calibrated $\mathrm{d}-\mathrm{c}$ supply, or the volt box and potentiometer could be replaced by an accurate digital voltmeter. 
A procedure often used in this test, and one that may be satisfactory in many cases is as follows: switch to AC-V (Fig. 3) and adjust the Lindeck potentiometer to balance; then switch to DC-V, adjust it to rebalance the Iindeck potentiometer and measure the $d-c$ voltage with the volt box and potentiometer. The procedure is then repeated with reversed $d-c$ voltage. The average of the two $d-c$ voltages should then equal the a-c voltage when corrections are applied for the volt box and potentiometer, and for any ac-dc difference in the transfer voltmeter.

However, as stated earlier, we very often have a drift in thermocouple output, mainly because of heating of the transfer voltmeter. Since we must make two exact adjustments after the AC-V is switched off (we must adjust the $d-c$ voltage and then measure $1 t$ ), an apparent but unreal difference between the two voltages can appear because of the drift. It is also quite difficult to determine just how much effect the drift is causing. If the $d-c$ adjustment and measurement require varying amounts of time, it may be difficult to obtain repeatable results.

The procedure suggested here will largely avoid the drift effect and may, at the same time, actually make the test a bit faster and less tedious. The potentiometer is set to a round-number value which is nominally equal to the $a-c$ voltage and the $d-c$ voltage is adjusted to balance the potentiometer at this setting. The Lindeck potentiometer is then adjusted to give a near mid-scale galvanometer reading (not necessarily a null reading). Three galvanometer readings are then made at time intervals which should be nearly equal: DC-V, AC-V and reversed $D C-V$. The $d-c$ voltage is adjusted to nominal value for each reading on $D C-V$. The difference between the $a-c$ reading and the average $d-c$ readings is a measure of the voltage difference. When converted to percent with the galvanometer calibration curve, it can be applied to the potentiometer reading to determine the a-c voltage, or it can be recorded as a correction to be applied to the a-c instrument under test-with due attention to sign (see sample calculation).

One precaution here may be worth mentioning. The leads between the volt box and ac-dc switch should be equal in resistance to those between this switch and the terminals of the test instrument to equalize the voltage drop in these leads. This can be important when working with low voltages.

The use of two galvanometers with spots on one scale (Fig. 3) 111ustrate an arrangement we have found to be convenient and efficient. If a stable a-c voltage standard is being tested at certain settings or if an a-c digital voltmeter under test is supplied from a stable source, then one observer, who has the d-c voltage controls at hand, can make the test. After preliminary setting of the potentiometer and Lindeck potentiometer, he sets the $\mathrm{DC}-\mathrm{V}---$ as indicated by a null reading on the top galvanometer spot---and reads the position of the spot on the bottom of the scale. A second reading on $\mathrm{AC}-\mathrm{V}$ and a third on reversed $\mathrm{DC}-\mathrm{V}$, each 
made after readjusting the input voltage to obtain a null on the top light spot complete a determination.

Figure 4 shows a similar setup for current measurements. The ammeter under test is set to the test point for the a-c reading and the $\mathrm{d}-\mathrm{c}$ current is adjusted to the same nominal value for the direct and reversed $d-c$ reading. Differences in galvanometer readings on $A C$ and the average for DC indicates the difference in the currents. The procedure is the same as before.

\section{PRECAUTIONS}

High accuracy can be obtained with transfer instruments only if the user is aware of certain limitations and takes appropriate precautions.

(1) In current measurements, it is usually better if the thermoelement is grounded. It should never be more than about one volt above ground. If the test ammeter must be grounded then the thermoelement can be put above ground by this smal1 potential, but we should maintain a minimum voltage across the insulating bead between the heater and thermocouple. For voltage measurements one end of the heater of the transfer voltmeter should always be connected to the \pm or ground

termina1. If binding posts rather than connectors are used, we must be careful to ground the proper terminal。

(2) The emf of a thermoelement decreases slightly for the first minute or two that it is energized, aside from any heating effect in shunts or series resistors. Therefore, it should have a minute or two to warm up. This is usually done anyway during the preliminary adjustments before the test is started; however, after any large change in the heater current we should wait a minute or two for the thermoelement output to stabilize. The multiplier in the voltmeter, as noted earlier, frequently drifts for some time: The temperature coefficient of the multiplier resistor is usually quite sma11, but even so, for highest accuracy, we should let it warm up until the drift is quite slow and then use a test procedure, such as that given in this paper, to minimize the effect of drifts on our determination.

(3) Since thermoelements have a frequency response which extends to $100 \mathrm{MHz}$ or more, they should be used in areas of reasonably low fleld strength. Interference from TV stations can be particularly troublesome with low-current thermoelements. A quick check of lead-borne interference is easily made by short-circuiting the input terminals, with the connections to the standard in place as in use, but with a11 power supplies off. There should be no change in indication of the galvanometer in the output circuit of the thermoelement. Such interference can often be avoided by using coaxial leads and shielded circuit components. In extreme cases, however, the instrument may have to be used in a shielded room. 
(4) We should be sure that our transfer instruments have small ac-dc differences and that they are known so corrections may be applied. They are usually negligible $(<0.005 \%)$ at mid-audio frequencies, but then increase (often as the square of the frequency) at the higher audio frequencies. They should be determined at the highest rated frequency-or at the higher frequency to be used---and at lower frequencies only as necessary。

Thermoelements also have a low frequency limit, below which they fall to integrate properly. The ac-dc difference may approach 0.02 percent at frequencies ranging from about $5 \mathrm{~Hz}$ for most 1 ow-range thermoelements to about $60 \mathrm{~Hz}$ for some thermoelements with ratings above 1 ampere. It is independent of the range for multirange converters in which a single thermoelement is used with shunts or multipliers. One ac-dc difference test (ordinarily at $20 \mathrm{~Hz}$ ) is recommended to verify the low-frequency accuracy.

(5) Replacing the thermoelement should not affect the ac-dc difference if other $a-c$ circuit components are not disturbed in such a way as to affect their reactance. Replacement thermoelements are generally available from the instrument manufacturer with the necessary characteristics and with negligible ac-dc difference.

\section{SAMPLE CALCULATIONS}

Galvanomecer Calibration

$\begin{array}{cccc}\text { TC emf } & 8.9 & 3.7 & \mathrm{mV} \\ \mathrm{V}_{1} & 100.00 & 60.0 & \text { volts } \\ \mathrm{V}_{2} & 101.00 & 61.2 & " \\ \mathrm{~V}_{1} & 100.00 & 60.00 & " \\ \% \Delta \mathrm{V} & 1 & 2 & \\ \mathrm{D}_{1} & 15.6 & 14.3 & \mathrm{~cm} \\ \mathrm{D}_{2} & 34.1 & 33.2 & " \\ \mathrm{D}_{1} & 15.8 & 14.5 & " \\ \triangle \mathrm{D} & 18.4 & 18.8 & " \\ \mathrm{~S} & 18.4 & 9.4 & \mathrm{~cm} / 1 \% \Delta \mathrm{V}\end{array}$

(Refer to curve - Fig, 2)

\section{Voltage Measurement}

Frequency $20 \mathrm{kHz}$

d-c volts set 75.000

TC mV 6.2

S $\quad 14.2$

$D_{\mathrm{dc}} \quad 24.1$

$\mathrm{D}_{\mathrm{ac}} \quad 26.2$

$D_{d c}($ rev.) 24.7

$\mathrm{D}_{\mathrm{ac}}{ }^{-\mathrm{D}} \mathrm{dc}+1.8$

(Positive sign indicates $A C$ is larger than DC)

$\frac{D_{a c}-D_{d c}}{S}=+0.13 \%$

$0.0013 \times 75=+0.098$ volts

$\mathrm{a}-\mathrm{c}$ Volts $=75.000$

$$
\begin{array}{r}
+0.098 \\
\hline 75.098
\end{array}
$$

If AC-DC Difference of the transfer voltmeter is $+0.02 \%$ at $20 \mathrm{kHz}, \quad a-c$ Volts $=75.113$ 


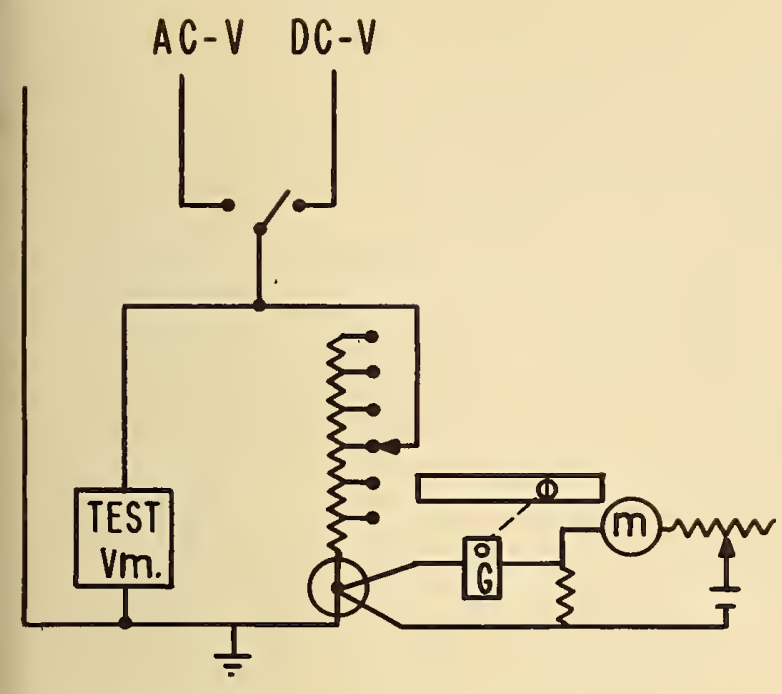

(a)

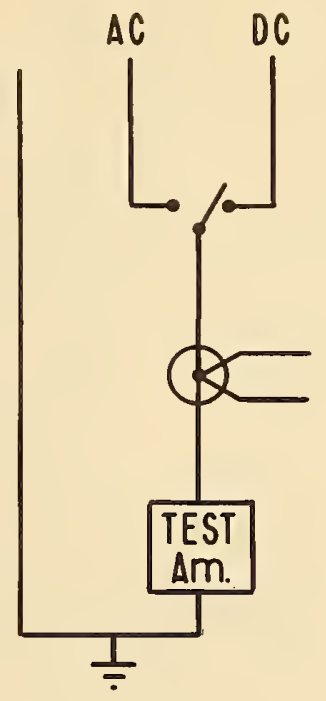

(b)

Fig. 1. Circuits for AC-DC Difference Measurements (a) for voltage, (b) for current
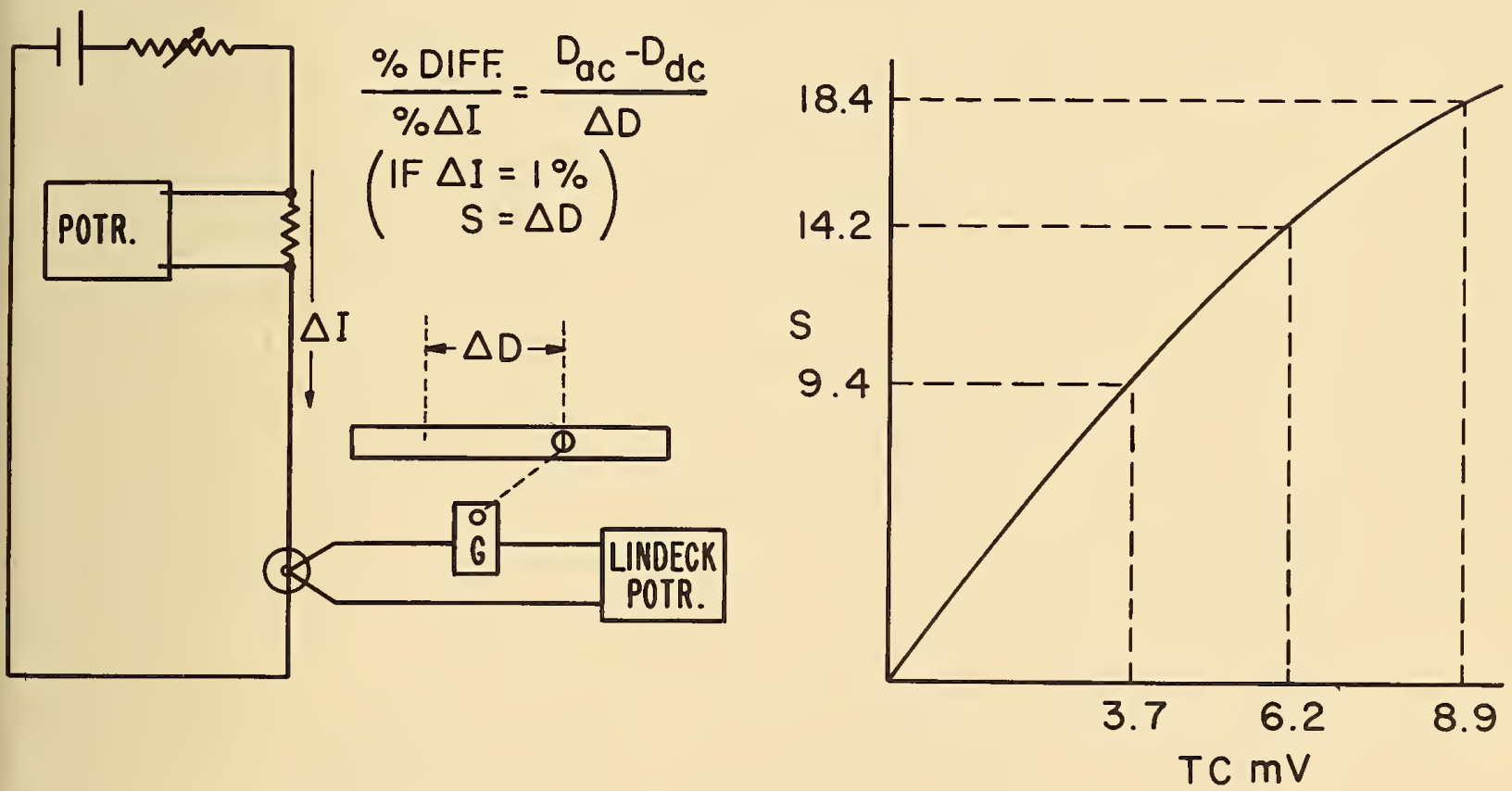

Fig. 2. Galvanometer Scale Calibration 


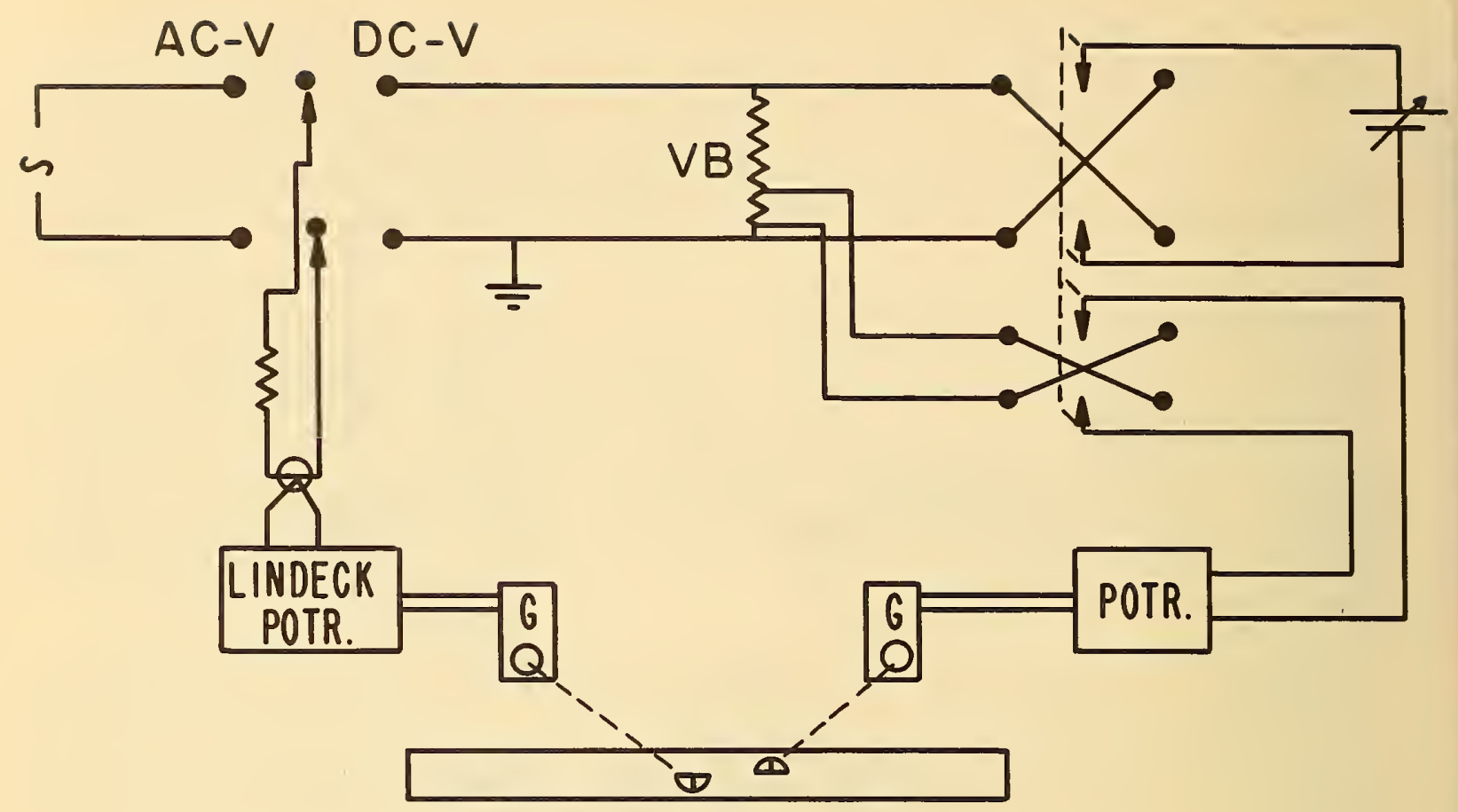

Fig. 3. Circuits for AC Voltage Measurements

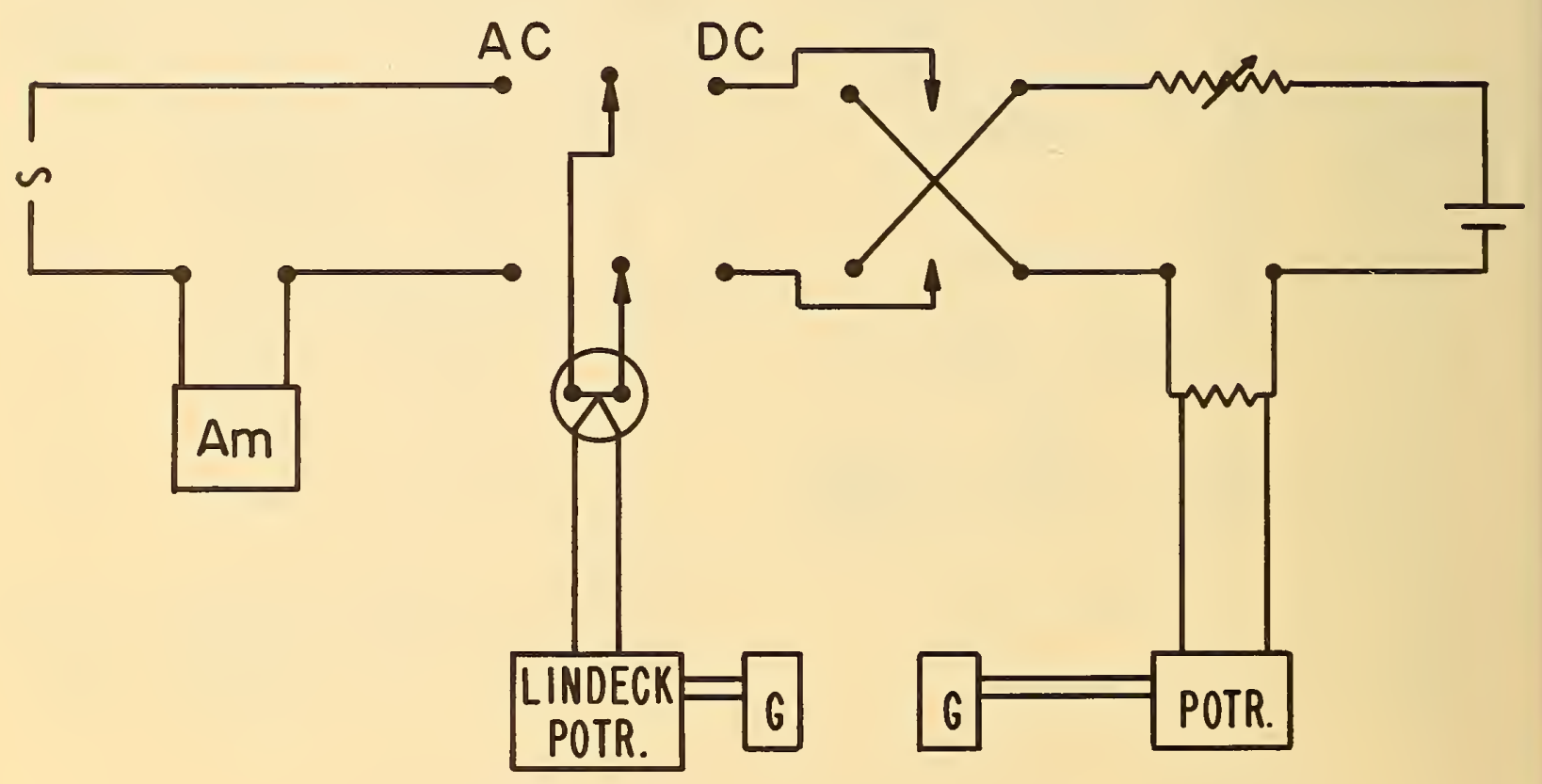

Fig. 4. Circuits for AC Current Measurements 

